\title{
Explosions from stellar collapse
}

\author{
Chris L. Fryer \\ Theoretical Astrophysics, Los Alamos National Laboratory, \\ T-6, MS B210, Los Alamos, NM 87545, USA
}

\begin{abstract}
The collapse of a massive star releases a considerable amount of gravitational potential energy. This energy is believed to be the power source of some of the largest explosions in the universe: supernovae, hypernovae, gammaray bursts. In this proceedings, we review the mechanisms by which the potential energy from stellar collapse can be tapped to produce these strong explosions, emphasizing how our understanding of massive stars can help constrain these mechanisms.
\end{abstract}

\section{Introduction}

Supernovae (SNe) and Gamma-Ray Bursts (GRBs) are among the strongest explosions in the universe. SNe inject $10^{51} \mathrm{erg}$ into their host galaxy with peak fluxes in excess of $10^{43} \mathrm{erg} \mathrm{s}^{-1}$, rivaling all the stellar emission in their host galaxy for a brief period. GRBs inject comparable energy $\left(10^{51-52} \mathrm{erg}\right)$ into narrow jets covering only $\sim 1 \%$ of the sky (Frail et al. 2001). These relativistic explosions produce strong bursts of gamma-ray emission. But these explosions have more in common than just being two of the largest cosmic fireworks. A large fraction of both these outbursts are believed to be powered by the collapse of massive stars. The gravitational potential energy released when the core of a massive star collapses to a neutron star (NS) or black hole (BH) is immense:

$$
E=\frac{\mathrm{G} M_{\text {core }}^{2}}{R_{\mathrm{NS}, \mathrm{BH}}}-\frac{\mathrm{G} M_{\text {core }}^{2}}{R_{\text {core }}} \approx 10^{54-55} \mathrm{erg}
$$

where $\mathrm{G}$ is the gravitational constant, $M_{\text {core }}$ and $R_{\text {core }}$ are, respectively, the mass and radius of the collapsing core, and $R_{\mathrm{NS}, \mathrm{BH}}$ is the NS,BH radius of the collapsed core.

Since the emergence of the concept of a 'star' composed of neutrons, scientists have believed that $\mathrm{SNe}$ could be powered by the collapse of a massive star down to a neutron star (Oppenheimer \& Snyder 1939). With observations of $\mathrm{Sk}-69^{\circ} 202$, the progenitor of SN 1987A, scientists obtained the first irrefutable proof that massive stars produce $\mathrm{SNe}$ (White \& Malin 1987). The 50-yr old idea that $\mathrm{SNe}$ are powered by the gravitational potential energy released during the collapse to a neutron star was vindicated by the detection of neutrinos from this explosion (Bionta et al. 1987; Hirata et al. 1987). Although the evidence for a stellar collapse mechanism is not so secure for GRBs, one of the leading models 
Table 1. Explosion mechanisms

\begin{tabular}{l|l|l}
\hline \hline mechanism & advantages & disadvantages \\
\hline \hline \multicolumn{3}{|c}{ SUPERNOVAE } \\
\hline $\begin{array}{l}\text { prompt explosion } \\
\text { neutrino driven } \\
\text { magnetic field driven }\end{array}$ & $\begin{array}{l}\text { physics straightforward } \\
\text { seems to work } \\
\text { explains asymmetries }\end{array}$ & $\begin{array}{l}\text { doesn't work with current EOS } \\
\text { detailed physics important } \\
\text { no quantitative studies, not enough energy }\end{array}$ \\
\hline \hline \multicolumn{1}{|c}{ HYPERNOVAE AND GAMMA-RAY BURSTS } \\
\hline $\begin{array}{l}\text { NS magnetic fields } \\
\text { magnetic collapsar } \\
\text { neutrino collapsar }\end{array}$ & $\begin{array}{l}\text { sufficient energy } \\
\text { quantitative studies }\end{array}$ & $\begin{array}{l}\text { no quantitative studies, not enough energy } \\
\text { no quantitative studies } \\
\text { barely enough energy }\end{array}$ \\
\hline \hline
\end{tabular}

for these powerful outbursts is the collapse of a massive star: the collapsar model (Woosley 1993; MacFadyen \& Woosley 1999).

To explain how these explosions occur, we must understand how the potential energy released during the collapse of a massive star is converted into kinetic energy. In this paper, let's review the mechanisms proposed to explain the powerful outbursts known as SNe and GRBs.

\section{Supernova mechanisms}

Three primary mechanisms have been proposed to extract the potential energy from core-collapse to produce supernova explosions: prompt explosions, neutrino driven explosions, and magnetic field explosions. All these mechanisms invoke the collapse of the iron core of a massive star down to a hot protoneutron star. Massive stars undergo an increasingly frantic existence until the iron cores in their centers collapse under increasing pressure. These cores are supported by thermal and electron degeneracy pressure. When the density and temperature get high enough, the iron atoms in the core are dissociated, effectively removing the thermal energy gained through years of fusion. In addition, at these high densities, electrons begin to capture onto protons, reducing the support of degenerate electrons. The loss of these pressure sources causes the core to contract, driving more iron dissociation and electron capture which leads to a runaway collapse (Figure 1). This collapse continues until nuclear forces and neutron degeneracy halt the collapse, that is, when the entire iron core has collapsed within $a \sim 100 \mathrm{~km}$ radius and infall velocities exceed 1/10th the speed of light!

\subsection{Prompt mechanism}

When nuclear forces and neutron degeneracy pressure halt collapse, the core bounces, sending a shock out through the star (Figure 1). Colgate \& Johnson (1960) proposed that this bounce could be the explosion. The advantage of such a model is its pure simplicity. Unfortunately, in most simulations, neutrino emission and dissociation of material as the shock moves out of the core sap the energy from the shock and cause it to stall. Only for specific equations of state (which are currently in disfavor) can explosions be produced (Baron, Cooperstein \& Kahana 1985; Baron et al. 1987). At this time, most SN theorists believe that this bounce shock stalls and must be revived to produce an explosion. 


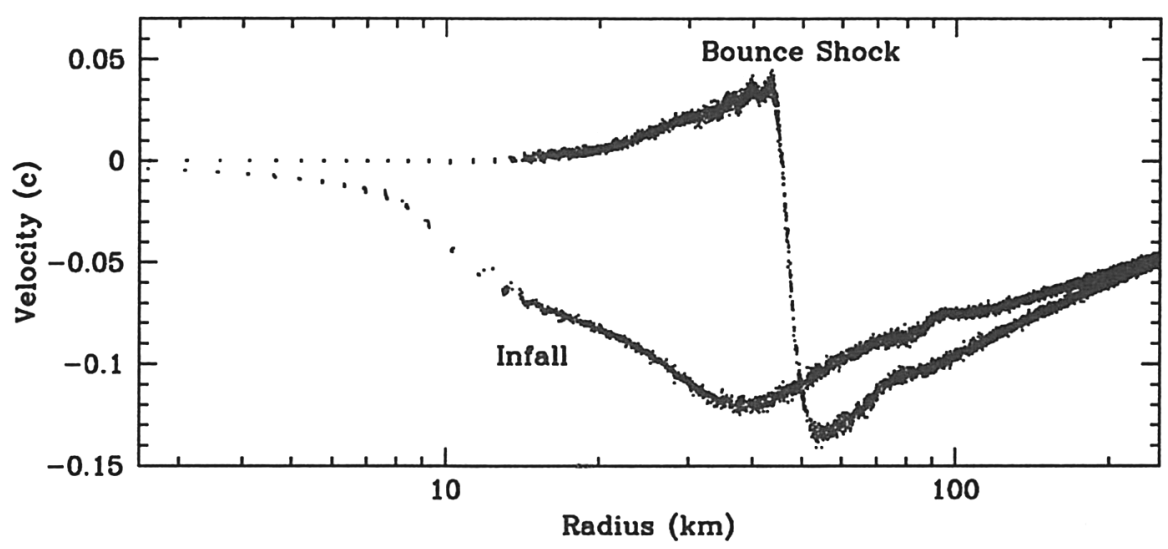

Figure 1. Velocity vs. radius plots of the collapse of a massive star before and just after the core reaches nuclear densities and bounces. This plots show a slice of the data from the 3-D simulations by Fryer \& Warren (2002).

\subsection{Neutrino driven mechanism}

An obvious way to revive the stalled shock is to tap into the enormous thermal energy of the collapsed core (also known as proto-neutron star). Due to the high densities, this energy leaks out of the core not through photon emission, but via neutrinos. Indeed, the densities are so high that even the neutrinos are trapped in the core and must diffuse out. Even so, the neutrino fluxes can exceed $\sim 10^{52} \mathrm{erg} \mathrm{s}^{-1}$. Colgate \& White (1966) proposed that neutrino deposition beyond the proto-neutron star would revive the shock, but over three decades of intensive research on this mechanism has yet to yield conclusive results. Colgate \& White (1966) simply assumed that a fraction of the neutrino energy (surely above $10 \%$ ) would be absorbed and drive the explosion. But detailed models of the core collapse have shown that converting even a few percent of the neutrino energy into explosion energy may not be easy.

The current paradigm for this model requires codes which must include the physics necessary to model the behavior of matter at nuclear densities, the accurate modeling of neutrino transport out of these dense cores, and the effects of convection just above (and maybe within) the proto-neutron star core (see Fryer 1999 for a review). The stall of the shock leaves behind a convectively unstable region on top of the proto-neutron star core. Convection can develop rapidly, especially if it is seeded by pertubations caused in Silicon and Oxygen shell flashes prior to collapse. Neutrinos from the hot proto-neutron star core deposit their energy into the lower part of this convective region, causing the convection to become even more vigorous (Figure 2). It is this convection that converts the thermal energy deposited by neutrinos into kinetic energy and ultimately drives an explosion. The star continues to collapse down on the convective region, and an explosion can not occur until the convective energy can overcome this cap produced by the infalling material. Models where this convection has been simulated in 2-D and 3-D now produce explosions (e.g., Fryer \& Warren 2002), 

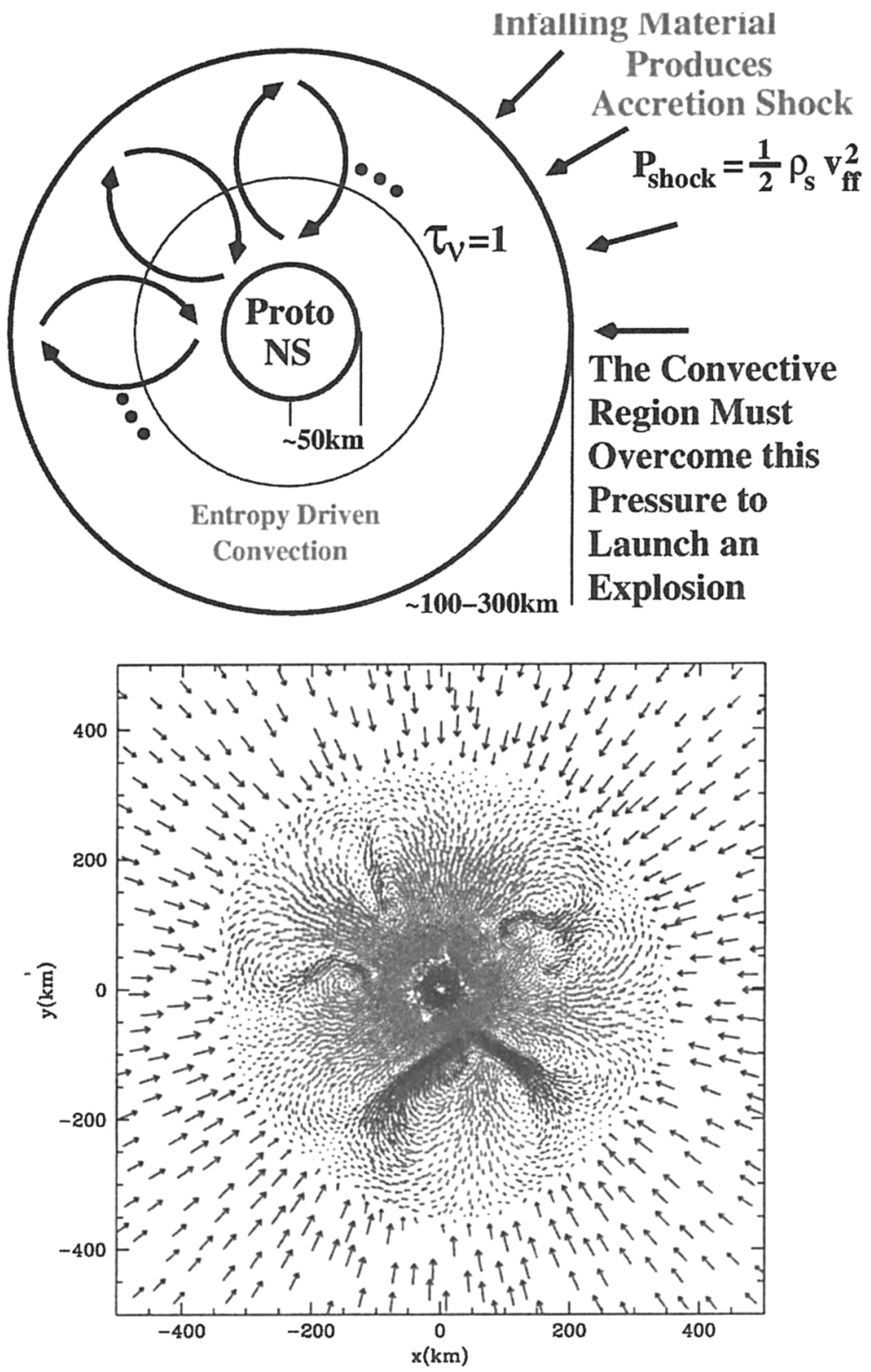

Figure 2. Upper panel: Diagram of the core of a massive star after bounce. In the center is the hot proto-neutron star above which is the convective region. Above the convective region, the rest of the star is falling down, forming a cap on the convective region. It is this cap that the neutrino driven pressure-cooker must overcome to drive an explosion. Lower panel: a slice of a 3-D simulation of this pressure-cooker (Fryer \& Warren 2002). 


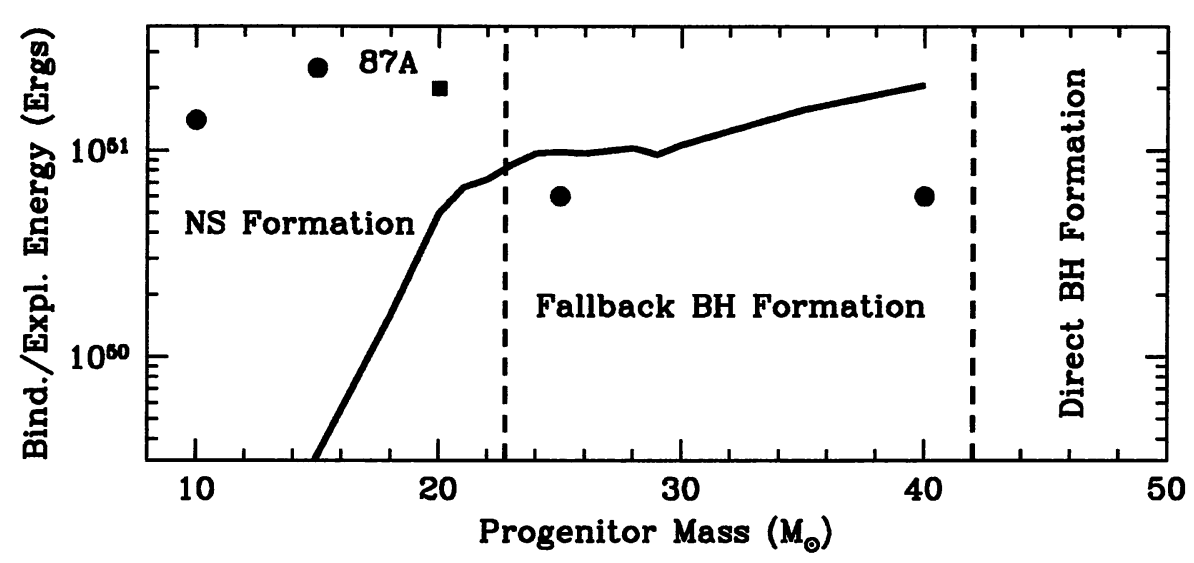

Figure 3. Binding energy/explosion energy vs. progenitor mass assuming no mass loss. The solid line is the binding energy of all but the inner $3 \mathrm{M}_{\odot}$ of the stellar core. If the explosion energy is not at least this powerful, the star will collapse to a black hole. The four circular dots denote the explosion energies from core-collapse simulations and the square is the predicted explosion energy observed from SN 1987A.

but bear in mind that the simple neutrino transport used in these models means that these results are far from conclusive.

Why is it so difficult to drive strong explosions? Bear in mind that some stellar collapses produce NSs and some produce BHs. To produce a $\mathrm{BH}$, a weak or no explosion must have occured. That is, the mechanism must have failed. It appears nature has conspired to place the supernova mechanism in a delicate batance where success or failure depends upon the details, so it is not surprising that changing minor details in the physics and progenitors of massive stars can change the entire fate of that star.

One of the factors that determines the fate of a massive star is the ram pressure of the infalling material that 'caps' the convective region. If this ram pressure is high, the energy in the convective region might not be enough to drive an explosion. A high ram pressure will eventually compress the convective region and cause the core to collapse to a black hole. If the ram pressure decreases quickly enough, the convective region can overcome this 'cap' and drive an explosion. This ram pressure is determined by the density structure of the stellar core which, in turn, depends upon the initial mass and mass loss of the progenitor star. Without mass-loss, the ram pressure increases with progenitor mass, making it harder to drive a supernova explosion with increasing progenitor mass (Fryer 1999). As the progenitor mass increases, the fate of the massive star changes from strong SN explosions with NS remnants to weak explosions with $\mathrm{BH}$ remnants formed via the fall back of matter, to the direct collapse to a black hole (Figure 3).

Mass loss complicates this picture even further. Fryer et al. (2002) found that the fate of a $60 \mathrm{M}_{\odot}$ massive star can change from a strong supernova leaving behind a NS remnant to no explosion at all with a $\mathrm{BH}$ remnant just by changing 
the mass loss by a factor of 3 ! Clearly understanding the life of massive stars makes a big difference in the fate these stars face when they end their lives.

\subsection{Magnetic fields}

Magnetic field models take advantage of the fact that as the star collapses, a portion of its potential energy is converted into rotational kinetic energy. A $10 \mathrm{~km}, 1.4 \mathrm{M}_{\odot}$ NS rotating with a period of $1 \mathrm{~ms}$ has a rotational energy of:

$$
=\frac{1}{2} \frac{2}{5} M_{\mathrm{NS}} R_{\mathrm{NS}}^{2} \Omega^{2} \approx 2 \times 10^{52} \mathrm{erg}
$$

where $M_{\mathrm{NS}}$ and $R_{\mathrm{NS}}$, and $\Omega=2 \pi /$ period are the mass, radius, and spin rate of the neutron star. Extracting only $10 \%$ of this energy could drive a reasonable SN explosion. LeBlanc \& Wilson (1970) suggested that for stars with high angular momenta, magnetic fields could be twisted by and extract energy from this rotation and drive axial jets. They assumed that the star was rotating so rapidly that it was centrifugally supported before it could collapse to a neutron star (many orders of magnitude above what is predicted today for the core rotation). Müller \& Hillebrandt (1979) found that for more modest (more realistic) rotations, extremely high magnetic fields ( $>10^{14-15}$ Gauss) were necessary to actually produce explosions. This effectively halted research in this field.

With the growing evidence of the connection between GRBs and SNe, Wheeler and collaborators (see Wheeler, Meier \& Wilson 2002 for a review) have revived the magnetic field model to drive SNe and Wheeler, Meier \& Wilson (2002) suggest a number of ways that magnetic fields could drive SN explosions. Note that magnetic fields provide a natural explanation for the large asymmetries observed in SNe (e.g., Höflich, these Proceedings). Unfortunately, all of these mechanisms still require large magnetic fields. Bolstered by the observations of magnetars and anomolous X-ray pulsars (Duncan \& Thompson 1992; Kaspi, Chakrabarty \& Steinberger 1999) which predict neutron stars with magnetic fields in the $10^{13-14}$ Gauss range, magnetic fields in excess of $10^{14-15}$ Gauss have begun to appear more reasonable. However, to make strong SN explosions, magnetic fields would have to be at the upper range of our limit $\left(>10^{15}\right.$ Gauss) and such magnetic fields have not been observed, even in the soft gamma-ray repeaters which are believed to arise from magnetars. High magnetic fields would not be buried after the SN explosion (Thompson \& Murray 2001), so if magnetic fields do produce SN explosions, they should be out there. Because the spindown rate of these highly magnetized neutron stars is high, there is a selection bias against detecting these neutron stars as pulsars, so we can not rule out the magnetic field SN mechanism based on the strong magnetic fields with current observations.

However, the rotation rates of massive stars may provide the killing blow for this mechanism. For the magnetic field mechanism to work, it must drive a SN explosion in the first second of collapse before the core collapses to a black hole. But in this first second, the proto-neutron star is hot and bloated, and it has not achieved the high angular momentum energy that we discussed above for a cold neutron star. Indeed, the explosion must occur when the proto-neutron star has a size of roughly $30-50 \mathrm{~km}$. At this size, a neutron star which will eventually produce a $1 \mathrm{~ms}$ pulsar will have an order of magnitude less rotational 
energy $\sim 10^{51} \mathrm{ergs}$ and to drive a strong supernova explosion, our magnetic field mechanism must be nearly $100 \%$ efficient. Worse still, it appears that, if anything, the evidence from massive stars is that stellar cores are rotating even less rapidly (see Heger, these Proceedings). With the stars Heger presented (with 5 times slower spin periods), the total rotational kinetic energy of the hot proto-neutron star is less than $10^{50} \mathrm{erg}$. If the rotational velocities in these stars is correct, the magnetic field mechanism can not work as the main mechanism for supernova explosions, although they may still contribute to the observed asymmetries. Understanding the rotation rates of massive stars is critical to the viability of this mechanism and the current evidence seems to disprove this mechanism.

\section{GRB and hypernovae engines}

The evidence that GRBs are somehow related to massive stars continues to grow. SN 1998bw (also known as GRB 980425) was the first strong evidence tying GRB and SN outbursts (Kippen et al. 1998). GRB 980425 was an exceptionally dim GRB and it was also a strange SN, and some have argued that the GRB/SN connection should not be made based on this evidence. SN 1998bw's estimated energy was much higher than normal SNe and its broad spectral lines are much better fit by asymmetric explosions (Nakamura et al. 1999). SN 1998bw marked the first of a new class of explosions called hypernovae or jet-driven supernovae (for the latest, see Mazzali et al. 2002).

But do these hypernovae produce normal gamma-ray bursts and what is their relation to SNe? Indirect evidence that GRBs are associated with massive stars has begun to grow: occurrence in star forming galaxies and regions (Fruchter et al. 1999), SN lightcurves popping above afterglow light curves (e.g., Bloom et al. 2002), growing evidence that some GRBs explode in wind-swept environments (Li \& Chevalier 2001). It may be that hypernovae are failed GRBs (ones that don't make relativistic shocks) or maybe hypernovae are GRBs not pointed in our direction, but in any event, it is likely that some GRBs and all hypernovae are associated with massive stars.

Three mechanisms have been proposed for hypernovae and long duration GRBs: magnetic fields off of a neutron star, magnetic fields around a black hole, neutrino annihilation above a black hole accretion disk. The latter two invoke the collapsar mechanism. Collapsars are massive stars that collapse to form BHs (either through fallback or direct collapse) that also have enough angular momentum to form an accretion disk around that BH. The potential energy released through the accretion in this disk (or possibly in the spin of the black hole for magnetic field mechanisms) can be extracted to produce a strong explosion. The structure of the massive star will focus this explosion into a narrow jet.

Although the collapsar mechanism doesn't need as much angular momentum as NS magnetic field mechanisms, a critical issue for collapsars to work is the amount of rotation in massive stars. Studies of massive stars clearly will help us understand these engines. It may be that some peculiar binary evolution is required to produced the high angular velocities needed to produce gammaray bursts. In addition, a $\mathrm{BH}$ must form to produce a collapsar and studies 
of mass-loss from massive stars will play a critical role in understanding $\mathrm{BH}$ formation.

\subsection{Neutron star magnetic fields}

This mechanism parallels the SN magnetic field mechanism and has its same foibles: not enough energy.

\subsection{Magnetic field collapsar}

Active galactic nuclei are believed to be powered by magnetic fields generated as these supermassive black holes accrete. Magnetic field mechanisms for GRBs and hypernovae are believed to be very similar to the mechanism that powers active galactic nuclei. The primary differences are that collapsar black holes are only a few solar masses in size versus the $>10^{6} \mathrm{M}_{\odot}$ black holes in active galactic nuclei and that the accretion rate through the disk is roughly $0.1 \mathrm{M}_{\odot} \mathrm{s}^{-1}$, many orders of magnitude greater than the accretion rates of active galactic nuclei. Both of these differences push toward much more energetic and rapidly varying outbursts than we observe in active galactic nuclei (see Popham, Woosley \& Fryer 1999, for a review).

A lot of papers have been published on magnetic field mechanisms for collapsars (Popham et al. 1999 has a list), but most boil down to a great deal of hand-waving and an efficiency factor at which magnetic fields convert the kinetic energy of the disk into explosion energy. Like Colgate \& White (1966), it seems reasonable to assume an efficiency factor of $10 \%$, but it may be another 30 decades before accurate quantitative calculations can be made. Bear in mind, that the true model for active galactic nuclei still hasn't been discovered, and magnetic fields remain difficult to model from first principles. If this mechanism powers GRBs and hypernovae, it may be a while before we understand it fully.

\subsection{Neutrino annihilation collapsar}

Neutrino annihilation is a more calculable engine for collapsars. Popham et al. (1999) studied this mechanism in some detail. The disks around these stellar mass black holes become dense and hot enough to emit copious neutrinos and anti-neutrinos of all flavors. The fluxes of neutrinos are so large that, in the region around the black hole, considerable annihilation between neutrino and anti-neutrinos can occur. This mechanism is not that efficient $(\sim 0.1 \%)$, but with the growing evidence that GRBs are beamed (Frail et al. 2001), the energy requirements for GRBs and hypernovae are within reach of neutrino-driven mechanisms. Although magnetic field mechanisms (with their association with active galactic nuclei) seem more plausible, neutrino annihilation is currently the only calculated mechanism for these outbursts, and this mechanism can produce (albeit barely) the energy necessary for long-duration GRBs!

Thus ends the review of the engines that produce SNe, GRBs, and hypernovae from stellar collapse. Table 1 summarizes these mechanisms, their advantages and problems. To understand these engines, we must understand their progenitors and how they evolve. In particular, the rotation and mass loss of massive stars are key uncertainties of our understanding of these powerful explosions. 
Acknowledgments. I'd like to thank T. Janka, A. Heger, and N. Langer for useful discussions on this topic. Much of this research was funded by a Feynman Fellowship and research grants at Los Alamos National Laboratory, a SciDAC grant for the Supernova Science Center, and Beomax Inc.

\section{References}

Baron, E., Cooperstein, J., Kahana, S. 1985, Phys. Rev. Letters 55, 126

Baron, E., Bethe, H.A., Brown, G.E., et al. 1987, Phys. Rev. Letters 59, 736

Bionta, R.M., Blewitt, G., Bratton, C.B., et al. 1987, Phys. Rev. Letters 58, 1494

Bloom, J.S., Kulkarni, S.R., Price, P.A., et al. 2002, ApJ (Letters) 572, L45

Colgate, S.A., Johnson, M.H. 1960, Phys. Rev. Letters 5, 235

Colgate, S.A., White, R.H. 1966, ApJ 143, 626

Duncan, R.C., Thompson, C. 1992, ApJ (Letters) 392, L9

Frail, D.A., Kulkarni, S.R., Sari, R., et al. 2001, ApJ (Letters) 562, L55

Fruchter, A.S., Thorsett, S.E., Metzger, M.R., et al. 1999, ApJ (Letters) 519, L13

Fryer, C.L. 1999, ApJ 522, 413

Fryer, C.L., Kalogera, V. 2001, ApJ 544, 548

Fryer, C.L., Warren, M.S. 2002, ApJ (Letters) 574, L65

Fryer, C.L., Heger, A., Langer, N., Wellstein, S. 2002, ApJ 578, 335

Hirata, K., Kajita, T., Koshiba, M., et al. 1987, Phys. Rev. Letters 58, 1490

Kaspi, V.M., Chakrabarty, D., Steinberger, J. 1999, ApJ (Letters) 525, L33

Kippen, R.M., Briggs, M.S., Kommers, J.M., et al. 1998, ApJ (Letters) 506, L27

LeBlanc, J.M., Wilson, J.R. 1970, ApJ 161, 541

Li, Z.-Y., Chevalier, R.A. 2001, ApJ 551, 940

MacFadyen, A.I., Woosley, S.E. 1999, ApJ 524, 262

Mazzali, P.A., Deng, J., Maeda, K., et al. 2002, ApJ (Letters) 572, L61

Müller, E., Hillebrandt, W. 1979, A\&A 80, 147

Nakamura, T., Mazzali, P.A., Nomoto, K., et al. 1999, Astron. Nach. 320, 4

Oppenheimer, J.R., Snyder, H. 1939, Phys. Rev. 56, 455

Popham, R., Woosley, S.E., Fryer, C.L. 1999, ApJ 518, 356

Thompson, C., Murray, N. 2001, ApJ 560, 339

Wheeler, J.C., Meier, D.L., Wilson, J.R. 2002, ApJ 568, 807

White, G.L., Malin, D.F. 1987, Nature 327, 36

Woosley, S.E. 1993, ApJ 405, 273

\section{Discussion}

WALBORN: (i) You have plotted SN 1987A at $20 \mathrm{M}_{\odot}$, but it had $16 \mathrm{M}_{\odot}$ at the explosion. The amount of mass lost depends on initial metallicity, and we know those parameters to some level for SN 1987A. They will be different in other systems. (ii) We also know how long it took the shock to reach the surface in SN 1987A. Can that be used as a constant in the appropriate model?

FrYer: (i) The initial mass determines the core mass. (ii) That time constrains the progenitor model, but since it was such a strange star (probably a binary) our theories are not at the stage that we can take advantage of this $\mathrm{SN}$ to constrain progenitor 
models.

ZINNECKER: You emphasized the importance of mass loss at the very end of your talk. Can you or someone in the audience convert your critical masses for non-masslosing supernova progenitor stars into the appropriate numbers for real mass-losing progenitor stars (depending on metallicity and rotation)? For example, what is the fate of a ZAMS $60 \mathrm{M}_{\odot}$ fast-rotating SMC single star?

FrYeR: Preliminary work to produce such conversions has been done (Fryer $\&$ Kalogera 2001; Fryer et al. 2002) but this is a difficult problem. We are working on a more complete study now, which will give our best estimates on the fate of massive stars as a function of mass, metallicity and rotation (Heger et al.). These results should be on the preprint server by the end of this year.

MAEDER: When there is a black hole formation, do all the onion skin layers collapse to the black hole or is there any mass cutoff above which some onion skin layers can escape from the black hole and contribute to the chemical yields?

FRYER: Without mass loss, stars above $40-50 \mathrm{M}_{\odot}$ will collapse directly to a black hole without any ejecta. Between $20-40 \mathrm{M}_{\odot}$, even though a black hole is formed, material is ejecta. Due to the large amount of mixing that is expected, quite a lot of $\mathrm{Ni}$ can be ejected even when large black holes are formed.

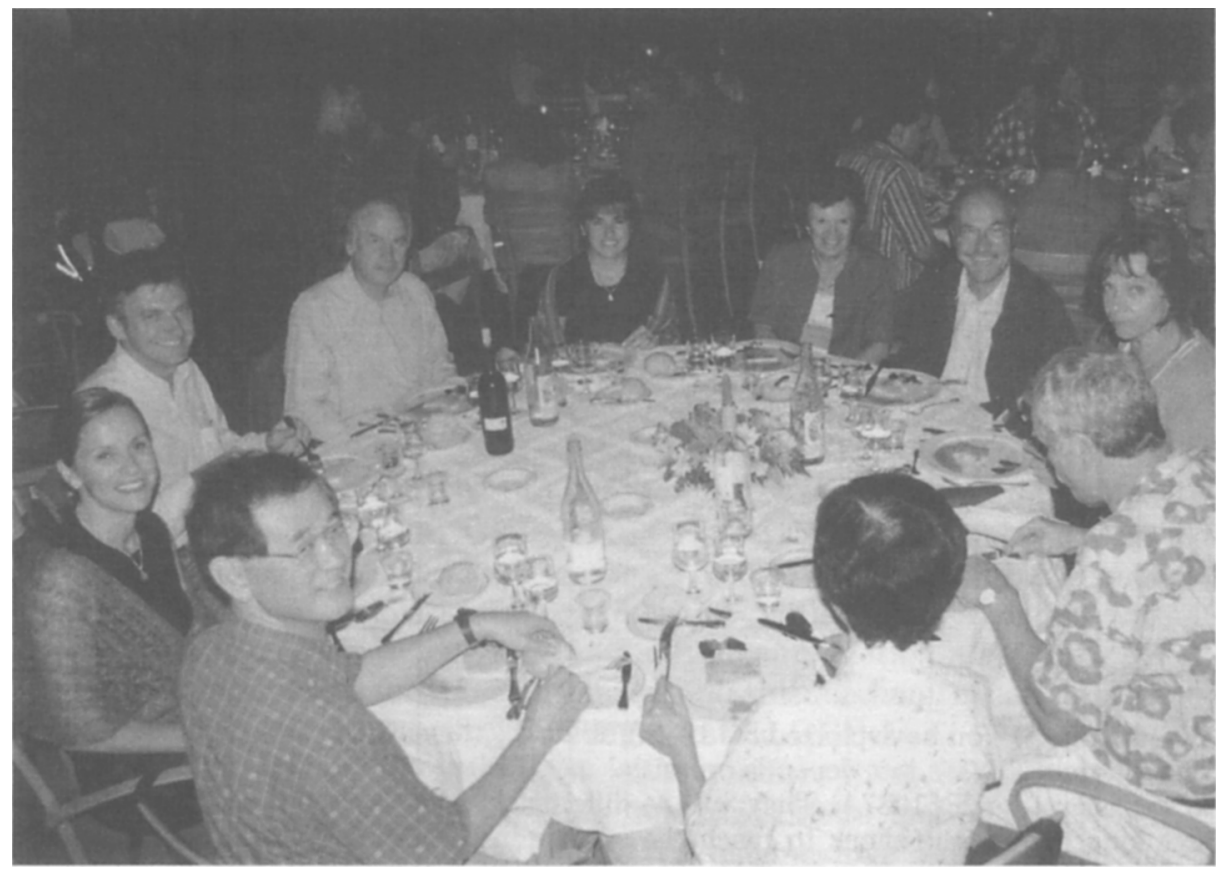

Spherical accretion. Clockwise: Mrs. Maeder, André Maeder, Mrs. Kudritzki, Rolf-Peter Kudritzki, Mrs. Nomoto, Ken Nomoto, Katia Cunha, Verne Smith, Wolfgang Gieren, and Mrs. Gieren 